\title{
Analysis of the Vagal Reflex Tracheal Constriction in the Dog
}

\author{
Tomokazu HOSOKAWA, Kazuhiro GOTO, Junzo KAMEl, \\ Kokichi OHTANI, Miwa MISAWA and Saizo YANAURA
}

Department of Pharmacology, School of Pharmacy, Hoshi University, 2-4-41 Ebara Shinagawa-ku. Tokyo 142, Japan

Accepted July 6, 1984

\begin{abstract}
We devised a preparation for measuring the vagal reflex tracheal constriction following the bronchoconstriction induced by histamine inhaled in the bronchial side in dogs. Properties of the vagal reflex tracheal constriction were investigated using this preparation. Histamine inhaled in the bronchial side caused the tracheal constriction following the bronchoconstriction. The tracheal constriction was inhibited by section of the bilateral superior laryngeal nerves or vagal cooling, respectively, but was not completely blocked. The combination of section of the bilateral superior laryngeal nerves and vagal cooling abolished the tracheal constriction. An i.v. administration of pentobarbital reduced both bronchoconstriction and tracheal constriction. These findings indicate that the tracheal constriction observed in the present study is mediated by the vagal refiex arc and that the extravagal pathway consisting of the recurrent and superior laryngeal nerves plays a role in a part of the afferent pathway of the vagal reflex airway responses. When the bronchoconstriction was completely abolished by isoproterenol inhaled in the bronchial side, the reflex tracheal constriction still existed. Transient inflation and deflation of the lungs caused reflex tracheal dilatation and constriction, respectively. We conclude that the vagal reflex airway constriction is due to complex effects which may be mediated by plural sensory receptors in the airways.
\end{abstract}

Present evidence indicates that the vagal reflex plays an important role in regulating airway smooth muscle. Several studies have reported the role of vagus nerves on the bronchoconstrictor responses to histamine $(1-6)$ and antigen (6-10). However, there are conflicting results about the degree of vagus nerve involvement. For example, Gold et al. (9) demonstrated that the vagal reflex was largely responsible for the increase in airway resistance following inhalation of antigen in allergic dogs, whereas Krell et al. (11) concluded that reflex mechanisms played only a minor role. In previous reports, we carried out a complete vagal blockade using the cooling technique in anesthetized dogs and demonstrated that the vagally mediated bronchoconstriction is a relatively major component of bronchoconstriction induced by intraarterial injection into the bronchial artery or inhalation into the airway of chemical mediators such as histamine, acetylcholine, serotonin and $\mathrm{PGF}_{2 \infty}$ (12), and Ascaris antigen (13).

Although the existence of the vagal reflex airway constriction in asthma was established, there are few investigations on the analysis of the vagal reflex airway response itself. There is therefore a need to devise a preparation which can sensitively and accurately detect the vagal reflex airway response itself. Thus, we attempted to detect the vagal reflex airway response using the in situ upper trachea perfusion preparation as described previously $(14,15)$. As a result, we devised a preparation for measuring the vagal reflex tracheal constriction following the bronchoconstriction induced by histamine inhalation in the bronchial side. Using this preparation, we investigated the properties of vagal reflex tracheal constriction. In addition, it was investigated how a change in 
peripheral airway mechanics affects the vagal reflex tracheal response.

\section{Materials and Methods}

Male mongrel dogs weighing between 10 and $13 \mathrm{~kg}$ were used. Light anesthesia was induced by the intramuscular injection of ketamine hydrochloride $(10 \mathrm{mg} / \mathrm{kg})$. The cervical trachea was exposed and transected at about $7 \mathrm{~cm}$ caudal to the larynx leaving the membranous wall intact. The membranous wall at the transected site was ligated with a thread to interrupt blood flow passage across the wall, while taking care not to disturb the recurrent laryngeal nerves. A tracheal cannula was inserted into the caudal site of the transected trachea. The animals breathed or were ventilated through this cannula. The left femoral artery was cannulated, and the surgical portion was treated with $1 \%$ procaine hydrochloride. The systemic arterial blood pressure was measured from the femoral arterial catheter using a pressure transducer (Nihon Kohden, MPU-0.5). The arterial pressure pulse was used to trigger a cardiotachometer, and the heart rate was continuously monitored (Fig. 1).

The animals were immobilized with decamethonium bromide (initial dose of $0.4 \mathrm{mg} / \mathrm{kg}$, i.v., and supplemental doses of $0.2 \mathrm{mg} / \mathrm{kg}$, i.v., every hour) and ventilated artificially with room air through the tracheal cannula connected to an artificial respirator (Natsume, KN-50) at a constant volume and a frequency of 20 breaths $/ \mathrm{min}$. End-tidal $\mathrm{CO}_{2}$ and $\mathrm{O}_{2}$ concentrations were continuously monitored by an expired gas monitor (SAN$\mathrm{EI}, 1 \mathrm{H} 21$ ) and were maintained at an optimal level of ventilation under resting conditions.

Responses of the bronchial musculature were measured by a modification of the Konzett-Rössler method (16). The lung was ventilated at a fixed volume of air under a constant pressure $\left(10 \mathrm{cmH}_{2} \mathrm{O}\right)$, and the ventilation overflow was measured using a pneumotachograph (Nihon Kohden, MFP1T) as an index of the change in airway resistance. The results were expressed as percent of the control level. Responses of the tracheal musculature were measured as changes in the intratracheal pressure of an air-filled balloon introduced into the rostral

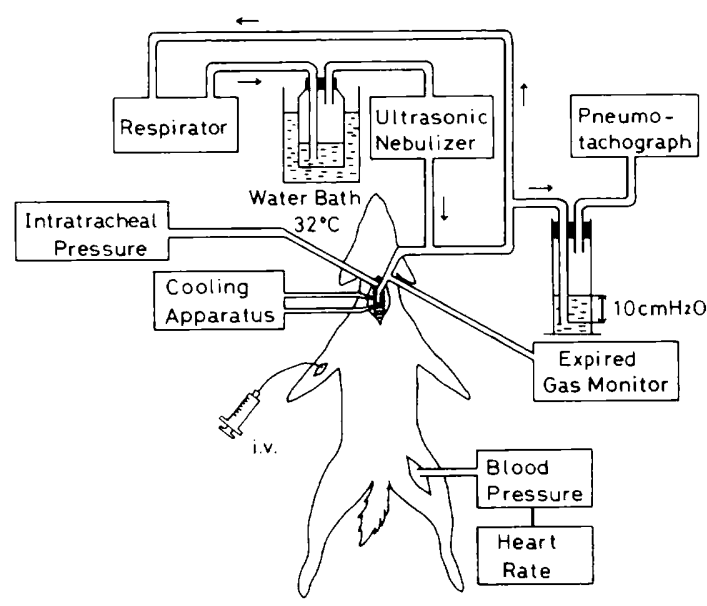

Fig. 1. Diagram of the preparation for drug inhalation to the airway arid for evaluating vagal reflex tracheal constriction in the dog. Tracheal musculature response was measured as a change in the intratracheal pressure of an air-filled balloon. Bronchial musculature response was measured as a change in ventilatior, overflow with a modification of the Konzett-Rössler method. The vagal cooling was performed by circulating cold water through the thermode attached to fit sriugly around the bilateral vagus nerves. Drugs were inhaled in the bronchial side using an ultrasonic rebulizer or injected i.v. into the cephalic vein.

side of the transected trachea. The air-filled balloon was connected to a pressure transducer (Nihon Kohden, LPU-0.1) through polyethylene tubing. The volume of air in the balloon was adjusted initially to give a resting intraluminal pressure of 50 $\mathrm{mmH}_{2} \mathrm{O}$. This pressure was found to be suitable for the observation of both constriction and dilatation of the tracheal musculature. Recordings were made on a polygraph (Nihon Kohden, RM-85). One hour was allowed for stabilization of the preparation after completion of the operation.

The vagal blockade was accomplished by cooling the bilateral cervical vagus nerves as described previously (12). The vagal cooling was performed by circulating cold water containing an anti-freeze liquid through the thermodes attached to fit snugly around the bilateral cervical vagus nerves. The thermodes were cooled to a temperature so that a thermister placed at the outflow port of the 
thermodes was kept at $0^{\circ} \mathrm{C}$. Drug inhalations were carried out for $10 \mathrm{~min}$ with an inhalant liquid volume of about $1 \mathrm{ml}$ in the bronchial side using an ultrasonic nebulizer (Nihon Kohden, TUR-3200) which was set into the circuit of artificial ventilation. In a few experiments, the lungs were inflated and deflated with measured volumes of air from a calibrated syringe.

Drugs used were histamine dihydrochloride (Wako Pure Chemicals), sodium pentobarbital (Tokyo Kasei) and isoproterenol hydrochloride (Sigma). All doses were expressed in terms of the base. All drugs were dissolved in saline solution.

The results shown in the figures are expressed as mean values \pm S.E. Statistical analyses were made using Student's $t$-test.

\section{Results}

Inhalation of saline solution in the bronchial side had no effect on the bronchial musculature and the parameters measured.

Typical recordings of the changes in ventilation overflow, intratracheal pressure, systemic blood pressure and heart rate by inhalation of a $0.00125 \%$ solution of histamine for $10 \mathrm{~min}$ in the bronchial side are shown in Fig. 2. Histamine inhalation caused a concentration-related increase in ventilation overflow, viz., bronchoconstriction, which
Was accompanied by increase in intratracheal pressure, viz., tracheal constriction (Figs. 2 and 3). The tracheal constriction occurred after a time lag following the bronchoconstriction. The amplitude of the tracheal constriction depended on the degree of the bronchoconstriction. The inhalation of histamine solution at the concentration used had no effect on the systemic blood pressure and heart rate.

The bronchoconstriction induced by $0.00125 \%$ histamine inhalation was un-

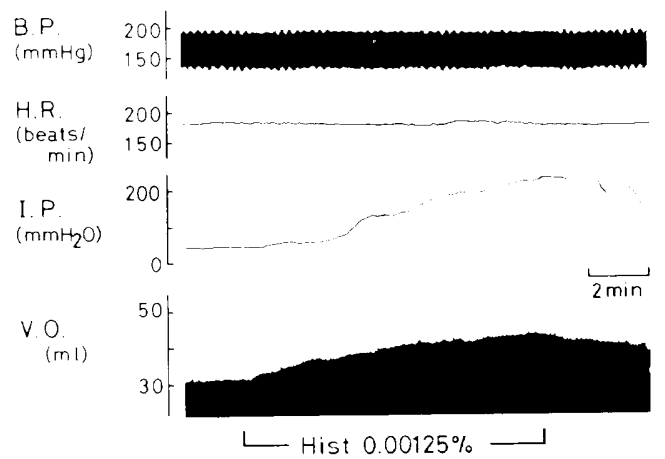

Fig. 2. Responses of systemic blood pressure (B.P.), heart rate (H.R.), intratracheal pressure (I.P.) and ventilation overflow (V.O.) to inhalation of histamine (Hist). A $0.00125 \%$ histamine solution was inhaled for $10 \mathrm{~min}$ in the bronchial side.
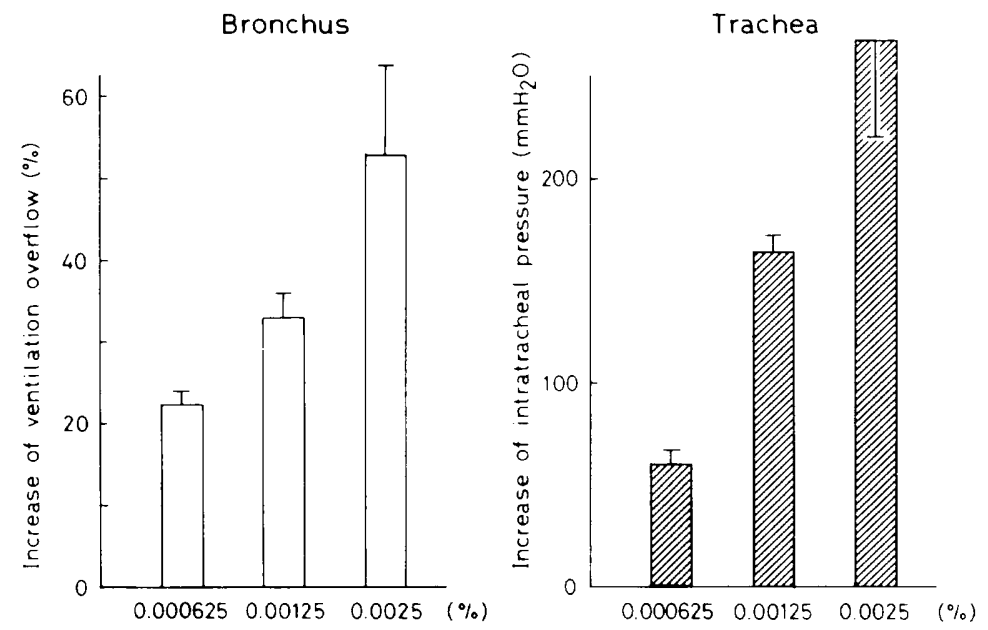

Fig. 3. Effects of histamine inhalation on the bronchial and tracheal musculature. Histamine solution inhaled for $10 \mathrm{~min}$ in the bronchial side caused a concentration-related bronchoconstriction which was accompanied by tracheal constriction. Each column is the mean value with S.E. for five experiments. 
affected by section of the bilateral superior taryngeal nerves, while the bilateral vagal cooling reduced the bronchoconstriction (Fig. 4, left panel). The combination of section of the bilateral superior laryngeal nerves and vagal cooling significantly inhibited the bronchoconstriction. On the other hand, the tracheal constriction following the bronchoconstriction was inhibited significantly by section of the bilateral superior laryngeal nerves and vagal cooling, respectively, but was not completely blocked. The combination of section of the bilateral superior laryngeal nerve and vagal cooling abolished
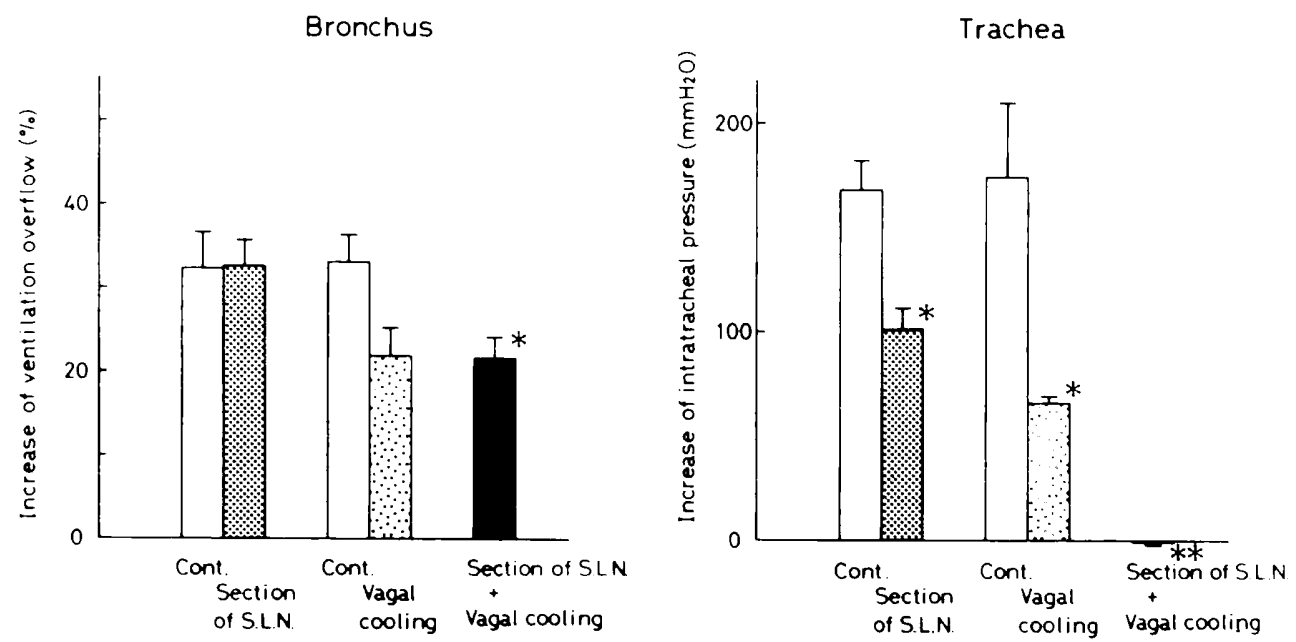

Fig. 4. Effects of vagal cooting and section of the bilateral superior laryngeal nerves (S.L.N.) on the bronchial and reflex tracheal constriction induced by histamine inhalation. A $0.00125 \%$ histamine solution was inhaled for $10 \mathrm{~m}$. $\mathrm{n}$ in the bronchial side. Each column is the mean value with S.E. for five to six experiments. The changes are sianificant at ${ }^{*} P<0.05$ and ${ }^{*}{ }^{*} P<0.01$ against control values.
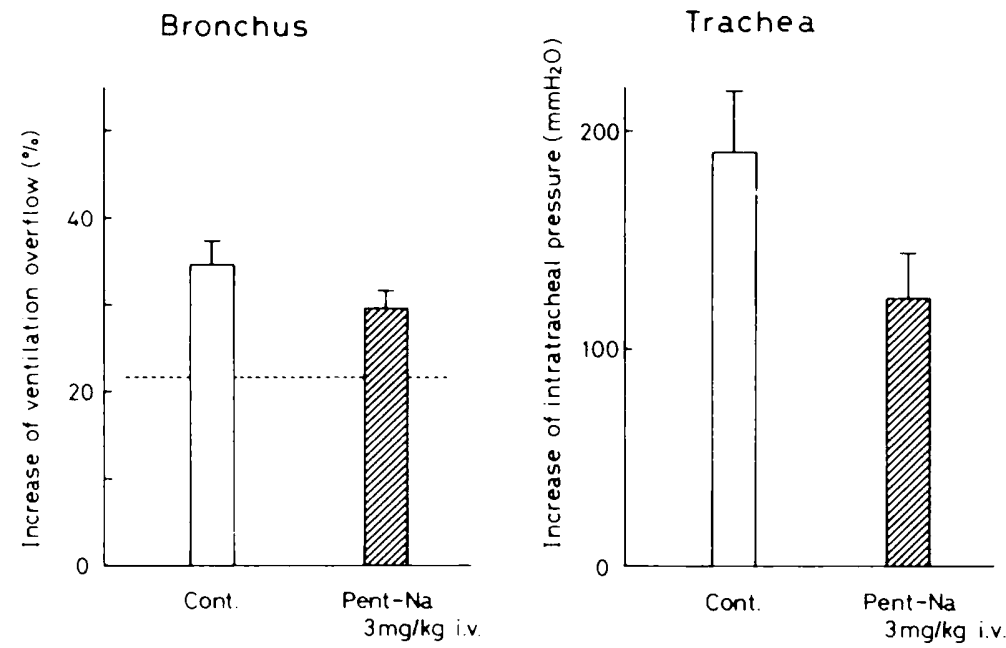

Fig. 5. Effects of pentobarbital sodium (Pent-Na) on the bronchial and reflex tracheal constriction induced by histamine inhalation. A $0.00125 \%$ histamine solution was inhaled for 10 min in the bronchial side. Each column is the mean value with S.E. for five experiments. Explanations for the dotted line (left panel) in the text. 

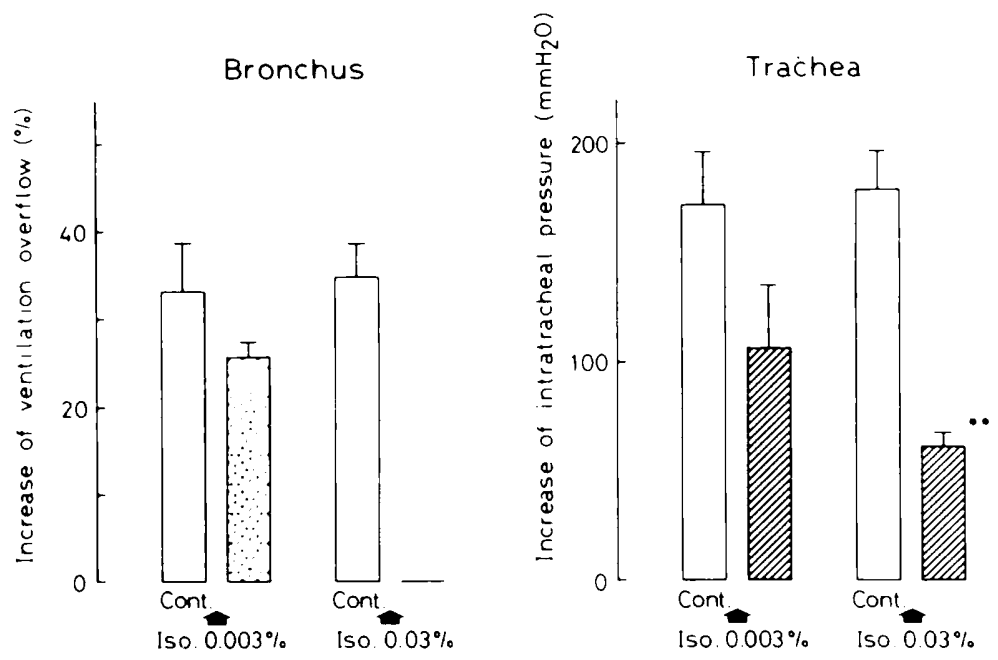

Fig. 6. Effects of isoproterenol (Iso) on the bronchial and reflex tracheal constriction induced by histamine inhalation. A $0.00125 \%$ histamine solution was inhaled for $10 \mathrm{~min}$ in the bronchial side. Isoproterenol inhalation was carried out in the brorichial side. Each column is the mean value with $S$. E. for five experiments. The change is significant at ${ }^{*} P<0.01$ against the control value.

the tracheal constriction (Fig. 4, right panel).

Pentobarbital in a dose of $3 \mathrm{mg} / \mathrm{kg}$ was injected i.v., 5 min before inhalation of histamine. An i.v. administration of pentobarbital at the dose used had no effect on the ventilation overflow, intratracheal pressure, systemic blood pressure and heart rate. Pentobarbital reduced bronchoconstriction induced by $0.00125 \%$ histamine inhalation. The tracheal constriction following bronchoconstriction was also reduced by pentobarbital (Fig. 5). The reduction of the tracheal constriction by pentobarbital was about $35 \%$ of the control response. The dotted line in the left panel of Fig. 5 shows the level of bronchoconstriction by histamine under blocking of superior laryngeal and vagus nerves (cf. left panel of Fig. 4), so that the responses above the dotted line may be a reflex bronchoconstriction. The reduction of the reflex bronchoconstriction by pentobarbital was about 38\%, and the reductive rate was similar to that of the tracheal constriction.

A concentration of $0.003 \%$ of isoproterenol inhaled in the bronchial side reduced the bronchoconstriction induced by $0.00125 \%$ histamine inhalation, and the tracheal constriction following the bronchoconstriction was also reduced. On the other hand, $0.03 \%$

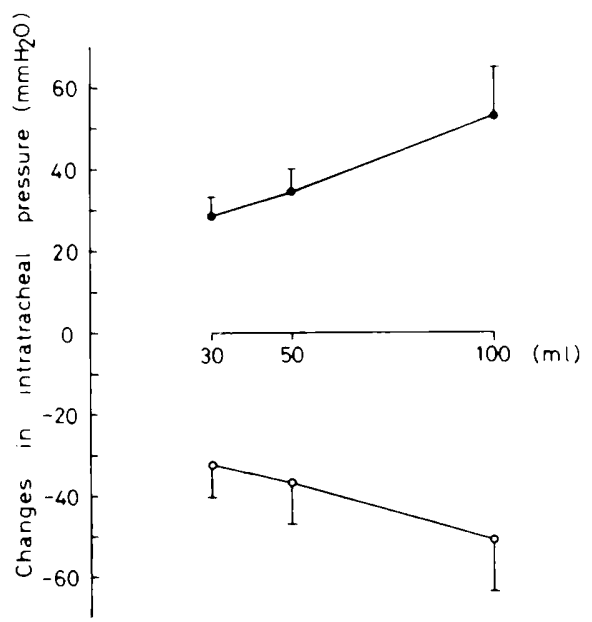

Fig. 7. Effects of lung inflation (_....) and deflation (-.) on the tracheal musculature. The lungs were inflated or deflated with 30,50 and $100 \mathrm{ml}$ of air from a caribrated syringe. Increasing inflation and deflation volume increased tracheal dilatation and constriction respectively. Each point is the mean value with S.E. for five experiments.

isoproterenol inhalation abolished completely the bronchoconstriction, and the tracheal constriction was inhibited but was not completely abolished (Fig. 6).

Results obtained with lung inflation and 
defiation on the reflex tracheal response are shown in Fig. 7. Transient inflation of the lungs in a volume of 30,50 and $100 \mathrm{ml}$ caused the tracheal dilatation. On the other hand, transient deflation of the lungs caused the tracheal constriction. The degrees of both tracheal dilatation and constriction depended on the inflation and deflation volume, respectively (Fig. 7). The tracheal responses evoked by inflating and deflating lungs were abolished by the bilateral vagal cooling.

\section{Discussion}

Recently, in anesthetized dogs, some investigators $(17,18)$ demonstrated a model of reflex tracheal constriction. In the present study, we attempted to detect the vagal reflex tracheal response in unanesthetized and immobilized dogs. As a result, histamine inhaled in the bronchial side caused the tracheal constriction following the bronchoconstriction. The preparation used was devised to interrupt blood flow between the upper trachea and bronchus (14). Furthermore, as shown in Fig. 2, histamine inhaled in the bronchial side had no effect on systemic blood pressure and heart rate. It is therefore suggested that the tracheal constriction which was observed here is not due to a direct effect of histamine inhaled in the bronchial side.

As shown in Fig. 4, a complete block of the tracheal constriction following the bronchoconstriction induced by histamine inhalation was observed by the combination of the vagal blockade and the section of the bilateral superior laryngeal nerves. Furthermore, the tracheal constriction was reduced by a low dose of pentobarbital. Jackson and Richards (19) demonstrated that a general anesthetic dose of pentobarbital inhibits the vagal component of histamine-induced bronchoconstriction by an action on the efferent pathway in the dog. On the other hand. Hukuhara (20) reported that the low dose of pentobarbital used in the present study shows an inhibitory action on neuronal discharges of the respiratory center without cardiovascular effects.

The above findings suggest that the tracheal constriction following the bronchoconstriction induced by histamine inhalation observed in the present study is mediated by a vagal reflex arc and that the present preparation is useful for investigating vagal reflex airway responses.

A complete block of reflex tracheal constriction was not observed by the bilateral vagal blockade alone. It is well known that afferent fibers exist in the superior laryngeal nerve. Several investigators have reported the existence of afferent fibers in the recurrent laryngeal nerve. Suzuki and Kirchner (21), in the paralyzed cat, recorded baroreceptor discharges from filament in the recurrent laryngeal nerve in which the nerve trunk was dissected between the electrode and larynx. Widdicombe (22) reported that the recurrent laryngeal nerve of the cat contains fibers from tracheobronchial endings. On the other hand, in the dog. Lemere (23) studied the fiber composition of the communicating branch between the superior and recurrent laryngeal nerves by histological methods and found it to contain sensory fibers. In the present study, it was found that the reflex tracheal constriction is completely blocked by the combination of the vagal blockade and the section of the superior laryngeal nerves. Vidruk (24) introduced the concept that an extravagal pathway consisting of the pararecurrent nerve (25), the ramus anastomoticus (23), the internal branch of the superior laryngeal nerve, and the superior laryngeal nerve carries afferent nerve fibers from sensory receptors. It may therefore be indicated that the extravagal pathway plays a role in a part of the afferent pathway of the vagal reflex airway responses. However. since the reflex tracheal responses evoked by inflating and deflating lungs were abolished by the bilateral vagal cooling alone, the involvement of an extravagal pathway in the vagal reflex airway responses may be related to the intensity of stimulation to the airway.

It has been suggested that a vagal reflex bronchoconstriction is elicited by stimulating irritant receptors $(3,5)$. In the present study, when the bronchoconstriction induced by histamine inhalation was reduced by $0.003 \%$ isoproterenol inhaled in the bronchial side, the reflex tracheal constriction was also reduced. Since Vidruk et al. (26) reported that isoproterenol had no effect on irritant 
receptor activity induced by histamine inhalation in dogs, the part of the reflex tracheal constriction reduced by $0.003 \%$ isoproterenol may be not due to stimulation of irritant receptors. On the other hand, when the bronchoconstriction was completely abolished by $0.003 \%$ isoproterenol, the reflex tracheal constriction was still in existence. The reflex tracheal constriction may be due to stimulation of irritant receptors. Therefore, the obtained findings indicate that the vagal reflex airway responses are mediated not only by the irritant receptors, but also by other receptors (i.e., stretch receptors). As shown in Fig. 7, transient inflation of the lungs caused vagal reflex tracheal dilatation. whereas transient deflation caused reflex tracheal constriction. Hering-Breuer inflation receptors are believed to cause bronchodilatation and are therefore unlikely to be the endings responsible for the histamine effect (27). Recently, Kaufman et al. (28) reported that in canine cardiovascular responses. slowly adapting receptors are likely to be responsible for the cardioacceleration evoked by inflation. However, the receptors related to lung deflation have yet to be demonstrated. Further work will be necessary to resolve these reflex responses which could represent a feed-back system controlling airway responses.

We conclude that the vaga! reflex airway constriction is due to complex effects which may be mediated by plural sensory receptors in airways.

Acknowledgment: This work was supported in part by Scientific Research Fund No. 5670090 from the Ministry of Education. Science and Culture of Japan.

\section{References}

1 Bouhuys, A., Jönsson, R., Lichtneckert, S., Lindell, S.-E., Lundgren, C., Lundin, G. and Ringquist, T.R.: Effects of histamine on pulmonary ventilation in man. Clin. Sci. 19, 79-94 (1960)

2 De Letona, J.M.L., De La Mata, R.C. and Aviado, D.M.: Local and reflex effects of bronchial arterial injection of drugs. J. Pharmacol. Exp. Ther. 133, 295-303 (1961)

3 DeKock, M.A., Nadal, J.A., Zwi, S., Colebatch, H.J.H. and Olsen, C.R.: New method for per- fusing bronchial arteries: Histamine bronchoconstriction and apnea. J. Appl. Physiol. 21, 185-194 (1966)

4 Simonsson, B.G., Jacobs, F.M. and Nadel, J.A.: Role of autonomic nervous system and the cough reflex in the increased responsiveness of arrways in patients with obstructive airway disease. J. Clin. Invest. 46, 1812-1818 (1967)

5 Karczewski, W. and Widdicombe, J.G.: The role of the vagus nerves in the respiratory and circulatory responses to intravenous histamine and pheny! diguanide in rabbits. J. Physiol. (Lond.) 201, 271-291 (1969)

6 Mills, J.E. and Widdicombe, J.G.: Role of the vagus nerves in anaphylaxis and histamineinduced bronchoconstrictions in guiriea-pigs. Br. J. Pharmacol. 39, 724-731 (1970)

7 Karzewski, W. and Widdicombe, J.G.: The role of the vagus nerves in the respiratory and circulatory reactions to anaphylaxis in rabbits. J. Physiol. (Lond.) 201, 293-304 (1969)

8 Yu, D.Y.C., Galant, S.P. and Gold, W.M.: Inhibition of antigen-iriduced bronchoconstriction by atropine in asthmatic patients. J. Appl. Physiol. 32, 823-828 (1972)

9 Gold, W.M., Kessler, G.-F. and Yu, D.Y.C.: Role of vagus nerves in experimental asthma in allergic dogs. J. Appl. Physiol. 33, 719-725 (1972)

10 Drazen, J.M. and Austen, K.F.: Pulmonary response to antigen infusion in the sensitized guinea pig: Modification by atropine. J. Appl. Physiol. 39, 916-919 (1975)

11 Krell, R.D., Chakrin, L.W. and Wardell, J.R., Jr.: The effect of cholinergic agents on a canine model of allergic asthma. J. Allergy Clin. immunol. 58, 19-30 (1976)

12 Yanaura, S., Goto, K., Kitagawa, H., Hosokawa, T., Misawa, M., Kobayashi, N. and Hayakawa, H.: Role of vagus nerves in bronchoconstriction induced by chemical mediators. Folia Pharmacol. Japon. 79, 571-579 (1982) (Abs. in English)

13 Yanaura, S., Mizuno, H., Goto, K., Kamei, J., Hosokawa, T., Ohtani, K. and Misawa, M.: Effects of 8 -(2-fluoroethyl)-3 $\alpha$-hydroxy- $1 \alpha \mathrm{H}$. $5 \alpha \mathrm{H}$-tropaniumbromide benzilate ( $\mathrm{Ba} 598 \mathrm{Br}$ ) on aliergy- and drug-induced asthmas. Japan. J. Pharmacol. 33, 971-982 (1983)

14 Yanaura, S., Hosokawa, T., Kitagawa, H. and Yamatake, Y.: Influence of tracheal muscular tone on the initiation of cough reflex. Japan. J. Pharmacol. 28, 447-455 (1978)

15 Yanaura, S., Hosokawa, T., Kitagawa, H. and Misawa, M.: Peripheral effects of morphine and 
codeine on the cough reflex. Japan. J. Pharmacol. 31, 529-536 (1981)

16 Konzett, $H$. and Rössler, R.: Versuchsanordnung zu Untersuchungen an der Bronchialmuskulatur. Arch. Exp. Pathol. Pharmakol. 195, 71-74 (1940)

17 Allott, C.P., Evans, D.P., Loveday, B.E. and Marshall, P.W.: A model of reflex tracheal constriction in the dog. Br. J. Pharmacol. 70, 419423 (1980)

18 Roberts, A.M., Kaufman, M.P., Baker, D.G., Brown, J.K., Coleridge, H.M. and Coleridge, J.C.G.: Reflex tracheal constriction induced by stimulation of bronchial $\mathrm{C}$-fibers in dogs. J. Appl. Physiol. 51, 485-493 (1981)

19 Jackson, D.M. and Richards, 1.M.: The effects of pentobarbitone and chloralose anaesthesia on the vagal component of bronchoconstriction produced by histamine aerosol in the anaesthetized dog. Br. J. Pharmacol. 61, 251-256 (1977)

20 Hukuhara, T., Jr.: Neuronal organization of the central respiratory mechanisms in the brain stem of the cat. Acta Neurobiol. Exp. 33, 219-244 (1973)

21 Suzuki, M. and Kirchner, J.A.: Laryngeal reflex pathways related to rate and rhythm of the heart. Ann. Otol. Rhinol. Laryngol. 76, 774-780 (1967)

22 Widdicombe, J.G.: Receptors in the trachea and bronchi of the cat. J. Physiol. (Lond.) 123, 71 104 (1954)

23 Lemere, F.: Innervation of the larynx. II. Ramus anastomoticus and ganglion cells of the superior laryngeal nerve. Anat. Rec. 54, 389-407 (1932)

24 Vidruk, E.H.: Extravagal innervation of canine tracheal stretch receptors. J. Physiol. (Lond.) 338, 11-20 (1983)

25 Lemere, F.: Innervatior, of the larynx. I. Innervation of laryngeal muscles. Am. J. Anat. 51, 417-437 (1932)

26 Vidruk, E.H., Hahn, H.L., Nadel, J.A. and Sampson, S.R.: Mechanisms by which histamine stimulates rapidly adapting receptors in dog lungs. J. Appl. Physiol. 43, 397-402 (1977)

27 Widdicombe, J.G. and Nadel, J.A.: Reflex effects of lung inflation on tracheal volume. J. Appl. Physiol. 18, 681-686 (1963)

28 Kaufman, M.P., Iwamoto, G.A., Ashton, J.H. and Cassidy, S.S.: Responses to inflation of vaga! afferents with endings in the lung of dogs. Circ. Res. 51, 525-531 (1982) 